\title{
Erratum
}

Arch Pharm Res Vol 34, No 11, 1773-1777, 2011

\section{Erratum to: SHINBARO, a New Herbal Medicine with Multifunctional Mechanism for Joint Disease: First Therapeutic Application for the Treatment of Osteoarthritis}

\author{
Sung-Yeol Lee', Hyoung-Keun Kwon ${ }^{1}$, and Sun-Mee Lee ${ }^{\mathbf{2}}$ \\ ${ }^{1}$ Green Cross, 303 Bojeong-Dong, Giheung-Gu, Yongin 446-770, Korea and ${ }^{2}$ School of Pharmacy, Sungkyunkwan Uni- \\ versity, Suwon 440-746, Korea
}

The Editors of the Archives of Pharmacal Research and the publisher hereby retract the article entitled "SHINBARO, a New Herbal Medicine with Multifunctional Mechanism for Joint Disease: First Therapeutic Application for the Treatment of Osteoarthritis" by Sung-Yeol Lee, Hyoung-Keun Kwon, and Sun-Mee Lee, which appeared in Arch Pharm Res, 2011, Vol. 34, No. 11, pp. 1773-1777, due to copyright issues of clinical data from Phase III trials (Figures 3 and 4).

The Editors of the Archives of Pharmacal Research, The Pharmaceutical Society of Korea 